\title{
Research of CWS' Particle Size Distribution based on Ultrasonic Attenuation Theory
}

\author{
WANG Weidong, ZHANG Chenglian, CHU Fengge \\ School of Chemical and Environmental Engineering \\ China University of Mining and Technology \\ Beijing, China \\ E-mail : wwd78816@sina.com
}

\begin{abstract}
- the key to reduce coal pollution is the development of clean coal technology and the improvement of the backward coal-burning technology. The coal water slurry (CWS) is the first substitute of the oil. The particle size distribution of CWS plays an important role in the quality control of CWS. Now there are three methods that are used to analysis the particle size distribution of CWS, screening method, settlement method, laser method. These methods produce some disadvantages when be used to forecast the distribution of CWS. Thus, this article proposes an ultrasonic method with effective medium theory model which can be accurately reflected in the acoustic attenuation characteristics of coal-water slurry based on structural average. Experimental simulation proved that effective medium model is fully capable of achieving on-line detection of coal-water slurry particle size, for detection of fine-and coarse-sized particle size distribution. Non-linear relationship between attenuation and particle size, the three-frequency method can be used to inverse calculation of its. Which we can achieve CWS granularity on-line, and continuously control the quality of CWS.
\end{abstract}

Index Terms - coal water slurry; effective medium model; size distribution; ultrasonic wave

\section{INTRODUCTION}

Coal is the most reliable energy, and not replaceable. Developing the advanced cleaning coal technology and transforming the backward coal-burning technology is the key to reduce the coal pollution. The CWS, coal liquefaction, coal gasification, coal-based methanol fuel and other advanced technologies using coal as an oil substitute fuel are the only way to convert coal into a kind of high-quality energy resources. The CWS is produced with physical method which is simple, low investment, low cost, and that is the first substitute of the oil [1-3].

The particle size distribution of coal-water slurry's particles plays a pivotal role on quality control of coalwater slurry. The particle size in coal water slurry requires not only the stipulation degree of fineness, but also good particle size distribution. Through the control of grain-size distribution, the high stack efficiency can be got, and which is a key technology to produce high density coal water slurry. And then the fast and accurate measure of particle size distribution of coal-water slurry is the most critical technical.

At present, the main methods of coal-water slurry particle size measurement are laser, sieve and sedimentation methods, etc. The above methods can only realize the off-line examination, and that are very difficult for real-time examination control grain-size distribution with above methods [4-8]. Therefore, it is very essential to introduce a new measuring technique ultrasonic absorption to realize the water-coal-slurry's granularity online examination. Application of ultrasonic absorption measurement to the determination of the size of particles suspended in a fluid is being given more and more attention. The major advantage of this technique resides in the fact that it is non destructive and can be employed with concentrated systems.

\section{THE EXISTING MEASURING METHOD ADVANTAGES AND DISADVANTAGES}

At present, a lot of methods are used to detect the particle size distribution of CWS, as follows: screening method, sedimentation method, laser particle size analysis.

\section{A. sieve method}

Sieve method is the oldest method which is commonly used to analysis the particle size. This method is wide used, and applied to the most significant industrial size range. With manual or mechanical vibration devices, the samples of known weight go through the smaller mesh screen one after another and the quantity on every screen is weighed, then through calculating the mass of each grain size percentage, the particle size distribution can be got.

The sieve method has the advantage of easy operation, low cost, application of more widespread. However, there are many obvious shortcomings about this method:

1) Through screening operation, the particles may be damaged or broken, causing measurement errors.

2) Except hard operation, noise, poor environmental conditions, the large sample volume, and because of the limited number of the used sieves, the results of accuracy is not good, especially for fine particles.

3) Long measuring time, complex operation, the result influenced by human factors are the disadvantages of this method. The longer the measuring time, the measurement results more detailed. Therefore, this operation time and operation method should have strict standards. 
4) Sieve method cannot achieve true weight distribution. More specifically, the sieve method is the measurement of two-dimensional size of the smallest particles, rod-like particles for the measurement error is significant.

\section{B. Sedimentation method}

Sedimentation method is that when particles in the gravity field and centrifugal force field subsidence. With the stokes settling velocity formulas, particle size distribution can be obtained by measuring the changes of settling velocity in the fluid medium, especially, in the liquid, thus the cumulative size distribution can be got. Sedimentation was the most popular size measurement method, the common instruments of sedimentation are pipette, sedimentation scale, sedimentation pipe, light through the gravity settling analyzer, the light through the centrifugal analyzer, X-ray scanning deposition apparatus, and so on.

This method is based on the basic principle of Stokes law: in the gravitational field, within a reasonable size, spherical particles in laminar flow conditions, the relationship between settling velocity and particle size can be presented as follows:

$$
V_{s}=\frac{\left(\rho_{s}-\rho_{f}\right) D^{2} g}{18 \eta}
$$

$V_{s}$ is the particle settling velocity; $D$ is the particle diameter; $\rho_{s}$ and $\rho_{f}$ are respectively the density of particles and sedimentation; $g$ is the acceleration of gravity, $\eta$ is the viscosity of the liquid.

Similarly, there are many obvious shortcomings about this method:

1) Slow measuring, the average measurement time is from 25 minutes to 1 hour, the long measurement time is more difficult to make repeated measurements, but also increases the chance of particle re-aggregation.

2) The temperature need to be constant during the measurement process. The dissolution medium viscosity coefficient changes with temperature, which directly affecting the measurement results.

3) The density cannot be measured for a mixtures of vary particles. Particle density must be known exactly before the measurement of particle size, which is a great disadvantage, because in many cases, for different coals, its density is different.

4) Dynamic measurement range is small. When the particle's radius is less than $2 \mu \mathrm{m}$, the Brownian motion is dominant; when larger than $50 \mu \mathrm{m}$, the sedimentation became violent and even the motion regularity of the particles is not in accordance with the Stokes law.

5) The measuring result is the Stokes diameter, or particle size with the equivalent settling velocity, which is equivalent sphere diameter with the same settling velocity. Thus the measurement of non-spherical particles must be modified, otherwise, the measuring result will be small.

\section{Laser method}

Laser particle size analyzer is a fast, accurate and noncontact particle size measuring instruments, which has been widely used in the chemical, energy, materials, environment, mining and many other engineering fields. Laser particle size analyzer mainly is based on two basic principles, one is the Fraunhofer diffraction theory, the other is the Mie light scattering theory.

The disadvantage of the method is that the measured material should be optically transparent, which requires coal-water slurry should be diluted to a certain measure the concentration. Therefore, it is difficult to achieve online measurement of coal water slurry. And this method is bad for narrow particle size distribution of the sample, the resolution is relatively low.

However, with the scientific and technological development and the requirements of production progress, more and high demands appear for measurement of particles. To solve these problems, the new technologies and particles instruments must be carried out. Therefore, ultrasonic detection technology came into being. The particles attenuation and particle size distribution can be got through measuring the attenuation of the ultrasonic wave energy. As sound' good penetrating, this method can be used to test high concentrations two-phase flow. Thus this method has incomparable superiority measurement for high concentration mixture. With a wide frequency range, the method of ultrasonic attenuation gives more attention to the researchers about the measurement of concentration and particle size distribution.

\section{AN EFFECTIVE PROPERTY OF CWS}

\section{A. The effective medium theory [EMT]}

At first, the spheroid with a radius $F$ should be constructed with a coal pellet inside and suspending liquid outside, which sphere immerses in water to form an effective whole, the effective properties of the whole is different from two phases and changes with the frequency shift, which including density, viscosity, adiabatic compression and so on.

In this method, we assume that the radius of a coal particle is a, and the center of another particle stands on $x_{1}$, then the overall average amplitude of the speed at the point $x$ can be expressed as $\langle\boldsymbol{u}\rangle\left(\mathrm{x} \mid \mathrm{x}_{1}\right)$, the average amplitude of the whole fields can be expressed as $\langle\boldsymbol{u}\rangle(\mathrm{x})$. With this method, the most critical question is to determine the effective sphere particle radius $R$.

As we know that the effective medium theory can be used to carry on the approximate calculation to the condition average field, but the prerequisite is that the measured pellet lies in the distant field, here the condition average field is equal to the unconditional average field, namely, when $\left|x-x_{1}\right| \rightarrow \infty$, then $\langle\boldsymbol{u}\rangle\left(\mathrm{x} \mid \mathrm{x}_{1}\right)=\langle\boldsymbol{u}\rangle(\mathrm{x})$, On the contrary, when $\left|x-x_{1}\right| \leq a$, the condition average field must satisfy the particles governing equation. 
Thus the simplest approximate calculation is that while $a \leq\left|x-x_{1}\right| \leq R$, the condition average equation satisfies the suspended fluid governing equation; while $\left|x-x_{1}\right| \geq R$, the average equation satisfies the unconditional average equation of the suspended fluid. The differences of different methods of the effective medium approximation lie in the choice of $\mathrm{R}$. If $\mathrm{R}=\mathrm{a}$, the merit is simple for calculation, the bad calculation result is the shortcoming; if $\mathrm{R}=\mathrm{a} \emptyset^{-1 / 3}$, which shows that the effective medium sphere is far from the particles in dilute suspension. The following formula is chosen in this paper:

$$
\frac{\phi R^{3}}{a^{3}}=1-L
$$

Where, $\phi$ is the volume concentration of coal slurry, $L$ is the structure factor of CWS, which can be detected by light scattering techniques. However, actually it can be got as follows with the Carnahan-Starling theory:

$$
L=\frac{(1-\phi)^{4}}{1+4 \phi+4 \phi^{2}-4 \phi^{3}+\phi^{4}}
$$

The effective medium radius $\mathrm{R}$ based on $\mathrm{L}$ fully includes the interactions between the majority of particles, as early as 1995, which is used by Dodd and Sangani and they found that,when R satisfy (1), the condition mean field play the correct leading role at the larger [9-13].

When the volume concentration is smaller, $\mathrm{L}$ can be expressed as $1-8 \phi+o\left(\phi^{2}\right), R \rightarrow 2 a$, therefore in good mixing dilute suspension, the effective medium starts in $2 a$, the fluid region starts in $a<r<2 a$. The equation $R=a \phi^{-1 / 3}$ shows that the effective medium began from the position which is far from the measured particle, this is unreasonable. When $\phi$ is smaller, $\mathrm{L}$ is also smaller, and that, $\mathrm{R} / \mathrm{a}=\circ\left(\varnothing^{-1 / 3}\right)$ is also given by (1). Thus the radius $\mathrm{R}$ based on (1) can give good prediction about the effective medium properties [14-16].

\section{B. Calculation of effective medium properties}

To calculate the attenuation and phase velocity of ultrasonic wave, the first thing need to be calculated is the effective properties of CWS. Assumed that the effective properties are same as that of pure liquid, thus the effective medium theory can be used to calculate the effective properties. Generally speaking, the calculation of the effective properties and attenuation follows the following steps: the first step is to calculate the effective properties $k_{s o}$ of suspending, the second step is the imaginary part and real part of $k_{\varepsilon e}$, the former is the sound attenuation, the latter is the ultrasonic speed $\frac{\omega}{C_{e 0}}$ [17-20].

\section{CONTROL SYSTEM}

\section{A. Measurement device}

Ultrasonic particle size testing process is usually as follows: Firstly, test the ultrasonic transducer transmits ultrasound wave with the signal device. Secondly, that wave gets through the examined media. For the impact of a variety of attenuation mechanisms, ultrasound intensity got from the receiver transducer is weakened. Thirdly, the particle size distribution information can be obtained with the ultrasonic attenuation model and also the corresponding inverse calculation of the particle size distribution.

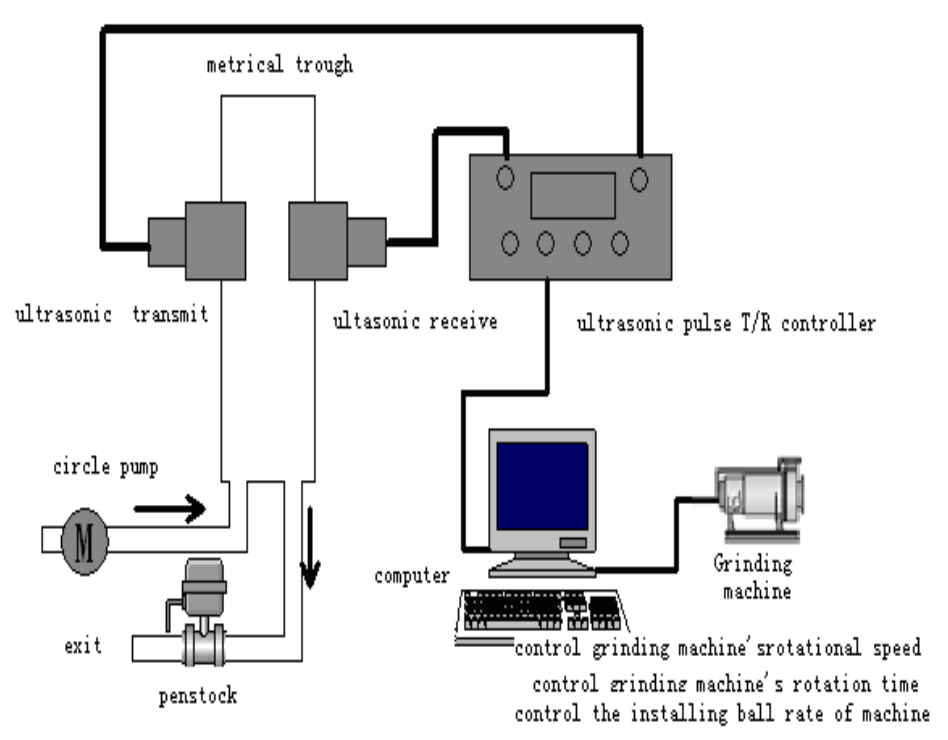

Fig.1 Sketch of Ultrasonic Particle Sizer Setup

CWS ultrasonic particle size testing device is shown in Fig.1, the entire system includes a measuring container, ultrasonic transmitter and ultrasonic receiver, ultrasonic pulse controller and data processing and display unit.

Moreover, the circulation dispersion system with a large volume and the temperature control system are designed respectively. Through enlarging the volume of sampling barrel, the full circulation of samples is realized, the representation is ensured, and disadvantage of present with low representation is overcome; On the other hand, the real-time temperature control system in the sample pond can increase the examination reliability by eliminating the influence of surroundings temperature.

\section{B. Sound attenuation measurement method}

The sound attenuation can be obtained through the ultrasound amplitude received in water and that of the CWS. So the formula of the sound attenuation coefficient is as follows.

$$
\alpha=\frac{20 \lg \left(A_{0} / A_{1}\right)}{L}
$$

This method is simple, and the reflecting loss does not need to be considered in the measuring process. 


\section{EXPERIMENTAL RESULTS AND ANALYSIS}

A. The relationship between the particle size and sound attenuation

1) The sound attenuation increases with the increase in concentration, and shows a non-linear relationship. When the particles size is larger than $125 \mu \mathrm{m}$ and the concentration smaller than $25 \%$, the sound attenuation increases slowly with the concentration, and when the particles size is less than $125 \mu \mathrm{m}$, the above concentration is about $10 \%$. But when the concentration is greater than $30 \%$, the sound attenuation increases significantly, regardless the radius of particles. The above phenomenon may be caused by the related scattering, which is brought by the stronger complex scattering and the interactions among particles with the increase of the concentration of coal particles and the radius of particles.

2) The relationship between sound attenuation and particles size of measured slurry is serious nonlinear, and there is a maximum value spot between $45 \sim 74$ micron, the minimum point at 110 micron, and then sound attenuation slowly increases as the grain size, thus the measurement of particles size distribution needs at least 3 frequencies for good results.

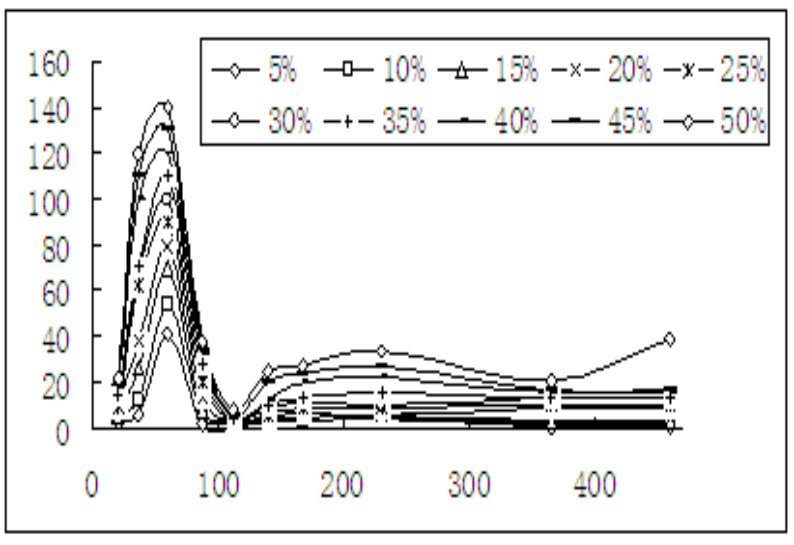

Fig. 2 the relation between sound attenuation and particles size

3) Under the same conditions, the sound attenuation increases with the frequency increase. At a concentration of less than $10 \%$, the rate of increase is small on sound attenuation, but along with density increase, the influence of frequency is obvious on the sound attenuation. This phenomenon's reason is also as a result of the change of sound damping action way, which is caused by the change of the action regional boundaries in the coal water slurry with frequency increase. On the other hand, the increase concentration will enhance the effects of the complex and relevant scattering.

\section{B. Factors of Affecting Velocity of slurry}

Fig. 3 shows that the sound attenuation and changes of velocity are simulated by the effective medium theory (EMT) model from different aspects in the coal water slurry. And the simulated values and measured values and other model predictions are compared, the result that EMT model can successfully forecast the sound attenuation and sound velocity for coal water slurry can be concluded. Two results as follows:

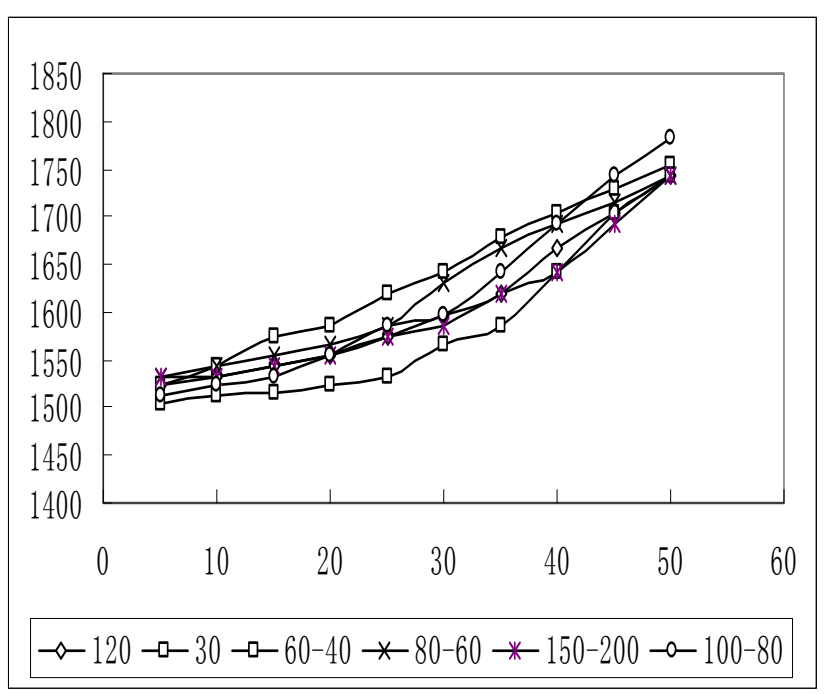

Fig.3 Variation of acoustic velocity with solid concentration in slurries of different particle sizes

1) In low concentrations $(<5 \%)$, the velocity change is little and irrelevant to the radius of coal particles, but the sound velocity increases significantly along with the increase of coal pellet concentration. In the same concentration, the speed of sound shows a downward trend along with the increase of the particle radius.

2) When the concentration of solid particles is greater than $40 \%$, the velocity and concentration show a nearly linear relationship, which provides a feasible approach for the density examination of highly concentrated watercoal-slurry.

\section{The relationship between the concentration of CWS and sound attenuation}

Fig4 shows the relation between sound attenuation with coal-water-mixture concentration

Sound attenuation increases as the concentration of coal-water-mixture, but the increasing relationship is nonlinear. When the particles' radius is large than 125 micrometer, the concentration less than $25 \%$, the acoustic attenuation's increase with the concentration is relatively slow; but when the particles' radius is less than 125 micrometer, the concentration decreases to be $10 \%$ or when the concentration is greater than $30 \%$, regardless of particle size, the acoustic attenuation rate will increase significantly.

Under the same conditions, the sound attenuation increases accordingly as the frequency increase. When the concentration of CWS is less than $10 \%$, the increase amplitude of sound attenuation is small, but the sound attenuation becomes obvious by frequency with the increase of CWS' concentration. This phenomenon is due to the change of the way of attenuation, with frequency increasing, the acoustic attenuation regional boundaries are changed in the coal slurry, thus, the sound attenuation caused by different ways increase. On the other hand, the 
increase of concentration will enhance the function of multiple scattering and related scattering.
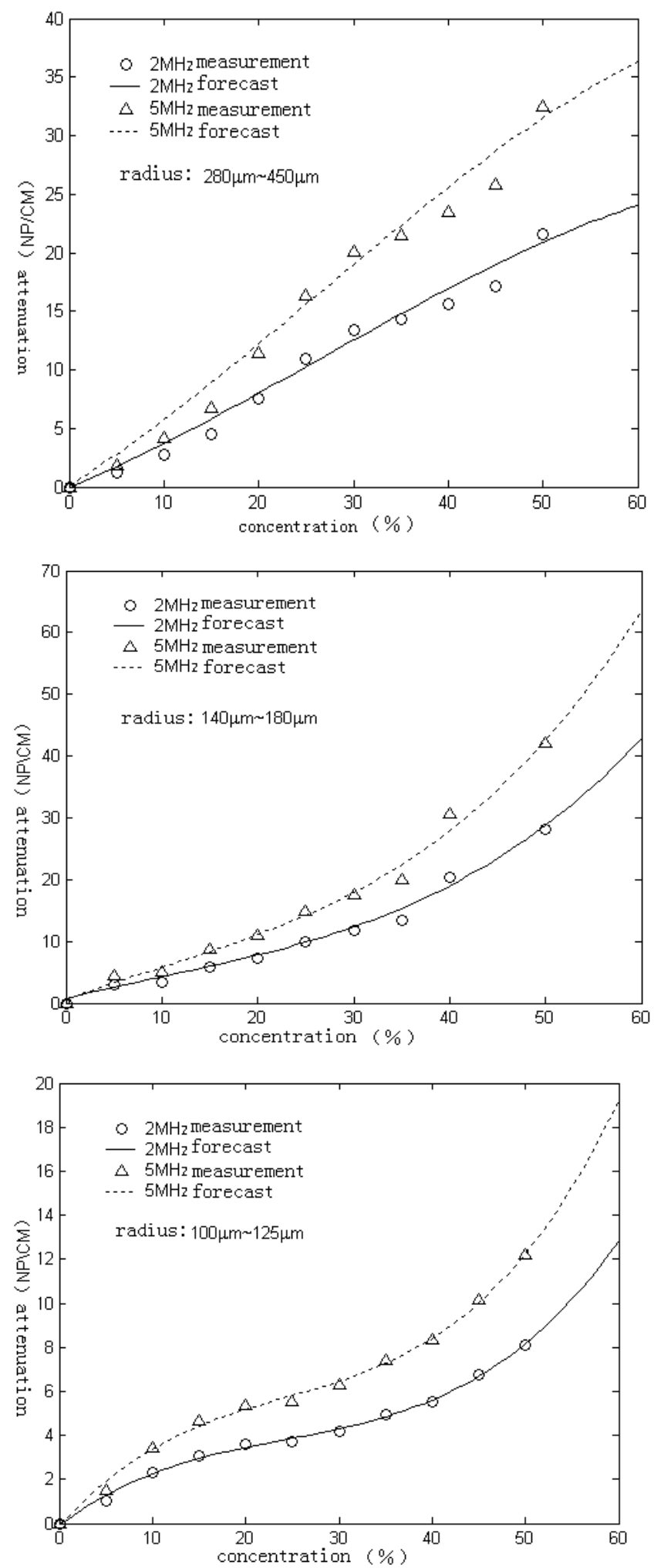
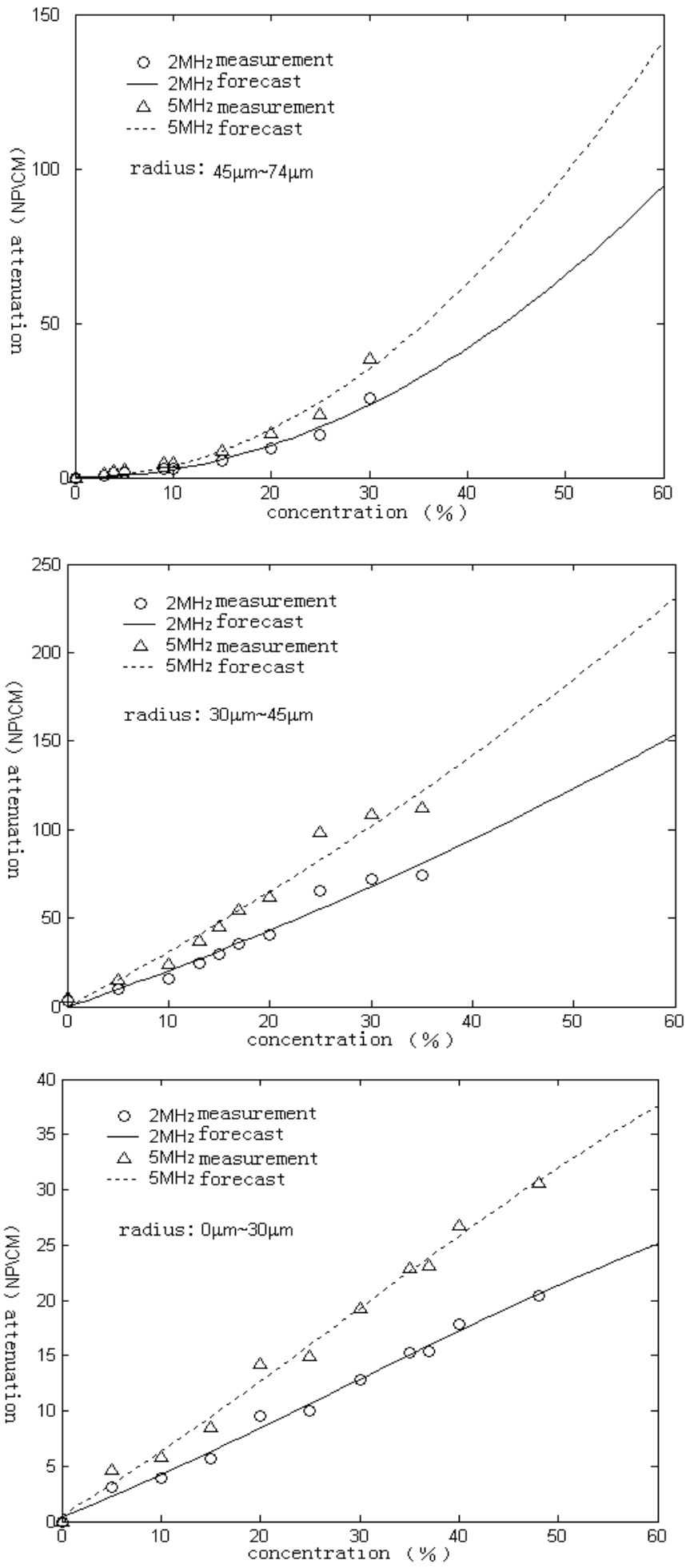

Fig.4 the relation between sound attenuation with coal-watermixture concentration

\section{The distribution curve}

Fig.4 shows the distribution curves of six grade particle system through the analog computation separately, we can conclude as follows:

1) In the more fine-grade, the result of ultrasound measurement accord with that of laser measurement. For the laser method is mainly used for a more accurate 
measurement of fine particles, ultrasound method can be used to measure smaller particles system.

2) In the coarse particle size, the result of ultrasound measurement varies greatly from the measurement result of laser method, while coincide with the measurement result of sieve. This may be because the upper limit of selected laser particle size analyzer is 300 microns, and the laser method is instable for large particles system, but sieving is more accurate for large particle size.
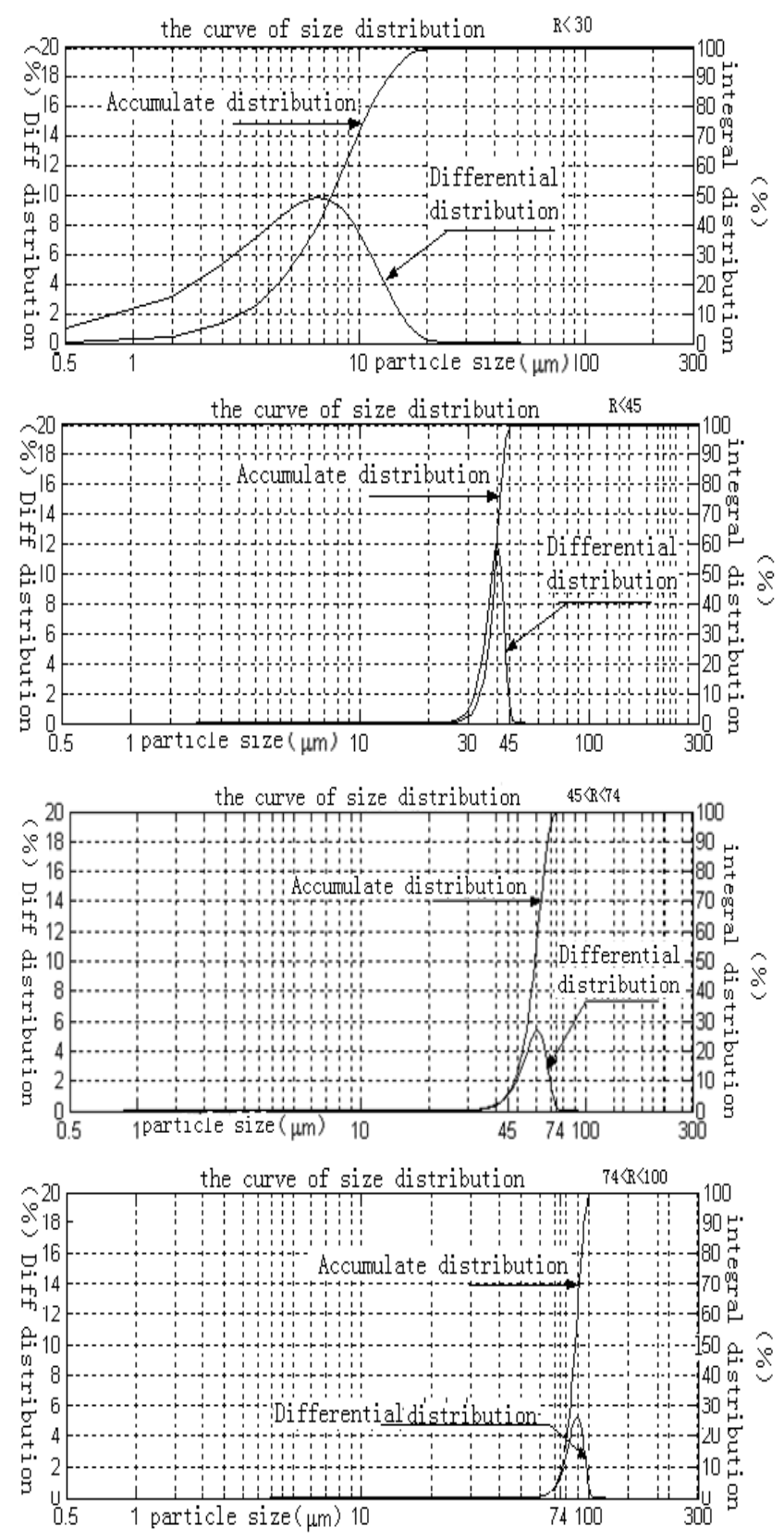
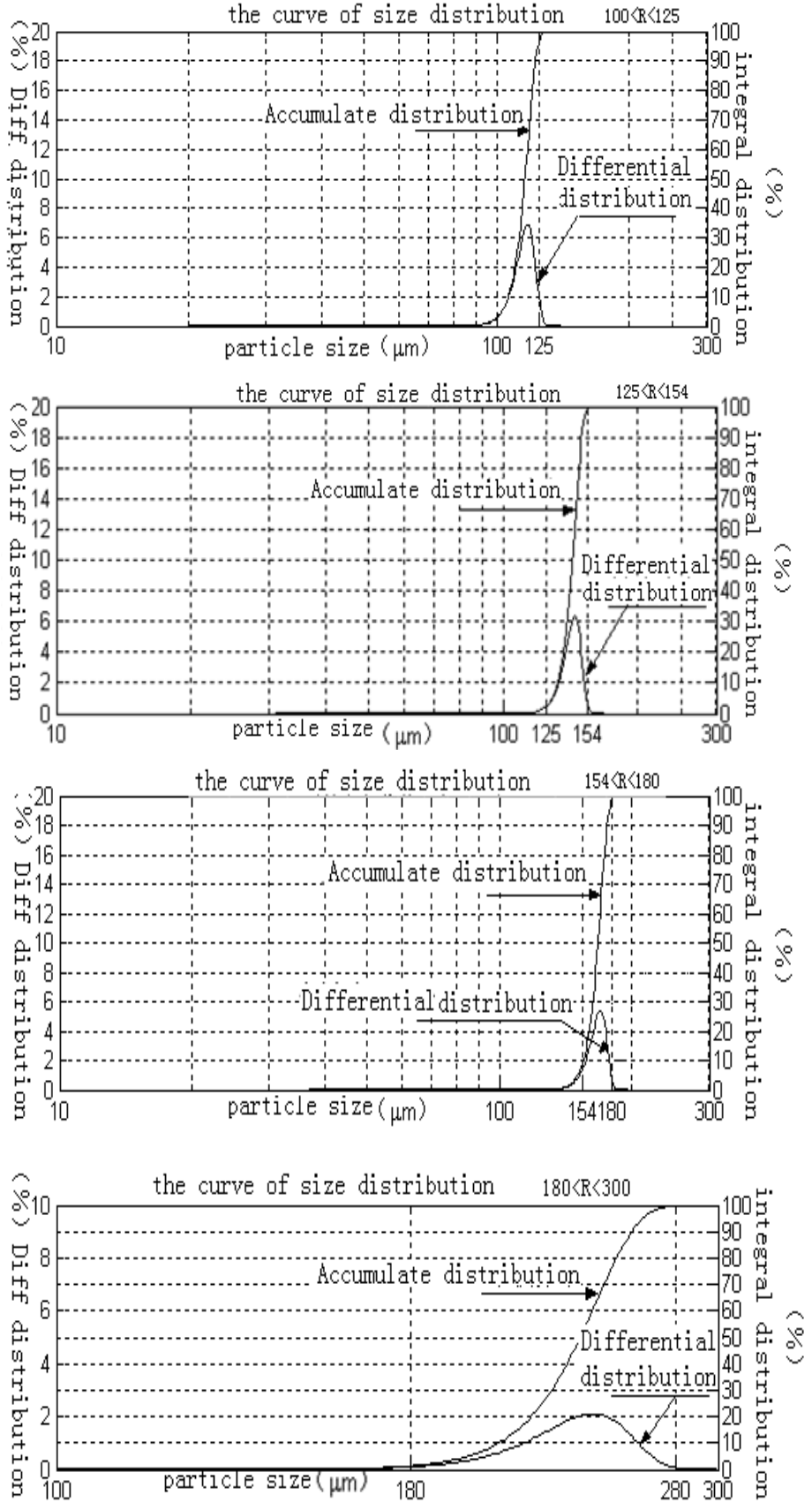

Fig. 5 Size distributions curves for different grade particle system

\section{CONCLUSIONS}

The above experimental and analysis show the following conclusions.

1) The measurement of CWS particle size distribution is entirely feasible, and the measurement range is wide with the effective medium theory.

2) A complete set of ultrasonic line monitoring system is designed based on above measurement principle, which design contains the temperature compensation and to eliminate air bubbles. 
3) The method of particle size distribution is compared with others, result shows that the effective medium theory can be used to detect the particle size distribution of CWS both coarse-size system and fine particles system ideally.

4) Though this method can be used to test particle distribution of CWS, but, this calculation progress is very hard, especially on computing technique and inversion algorithm. On these aspects, the author does not give a detailed description.

\section{ACKNOWLEDGMENT}

Financial support provided by the youth fund of China University of Mining and technology, Beijing (Project 2009QH04) for this work is gratefully acknowledged.

\section{REFERENCES}

[1] Zhang Rongzeng. CWM Preparation of [M]. Beijing: Science Press, 1996.(in Chinese)

[2] Wang Chuan-Jian, Hao and India. CWS on behalf of the oil inspection report. A study tour for Ministry of Coal Industry Report, 1985. (in Chinese)

[3] Su MingXu, Cai Xiaoshu, Huang Chunyan et al. Ultrasonic attenuation measurement of particle size [J]. Instrument Technology (Supplement). 2004,25 (4) :1-2. (in Chinese)

[4] T. Allen. Particle size determination [M]. China Building Industry Press, 1984 (8)

[5] Suming $\mathrm{Xu}$, Cai Xiaoshu, Xu Feng, et al. Measurement of ultrasonic attenuation in suspension of particle size and concentration [J]. Acoustics. 2004,29 (5) :440-444.

[6] He Guichun. Ultrasonic slurry particle size measurement of nonlinear modeling of [D]. Beijing: Beijing University of Technology, 2006. (in Chinese)

[7] Su Mingxu. Particle two-phase medium particle size and concentration of ultrasonic measurement theory [D]. Shanghai: Shanghai University of Technology, 2002. (in Chinese)

[8] Epstein P S, Carhart R R. The Absorption of Sound in Suspensions and Emulsions: I. Water Fog in Air.J.Acoust [J].Soc.Am.1953, 25:553 565.

[9] Waterman P C,Truell R. Multiple Scattering of waves [J]. J. Math. Phys. 1961, 2:512 540.

[10] Allegra J R, Hawley S A. Attenuation of Sound in Suspensions and Emulsions: Theory and Experiments [J]. J. Acoust. Soc.Am.1972, 51:1545 1564.

[11] Y. Hemar, li. Herrmann, P. LemarAchal, R. Hocquart and F, Lequeux.Effective Medium Model for Ultrasonic Attenuation Due to the Thermo-Elastic Eftect in Concentrated Emulsions [J].J. Phys. II Fance 7 1997.637 647.

[12] L. L. Foldy.the multiple scattering of waves, Phys. Rev. 1945, 67, 107 119.

[13] P C. Waterman and R Truell. multiple scattering of waves, J. Math.Phys. 2, 512-537 1961.

[14] P. Lloyd and M. V. Berry, Wave propagation through an assembly of spheres. IV. Relations between different multiple scattering theories, Proc. Phys. Soc. London 91, 678 688, 1967.

[15] .C.Waterman and Rohn Truell. Multiple Scattering of Waves [J]. J. Mathematical Physics, 1961, 2:513 540.

[16] L. L. Foldy.The multiple scattering of waves. I. General theory of isotropic scattering by randomly distributed scatterers [J]. Phys. Rev. 67, 107 119, 1945.

[17] XIA Yuming, WANG Weidong, and XU Zhiqiang, "Numerical computation of laminar flow pipeline transport axial flow field," 2009 International Conference on Information Technology and Computer Science (ICITCS 2009), 2009, pp. 196-199. (in Chinese)

[18] XIA Yuming, WANG Weidong, TIAN Jinyun, XU Zhiqiang, "Computational error of laminar flow pipeline transport axial flow field," 2009 International Conference on Computer Science and Information Technology (ICCSIT 2009), 2009, pp. 635-639.
[19] U Riebel. The fundamentals of particle size analysis by means of ultrasonic spectrometry [J].Particle and Particle Systems Characterisation, 1989, 6:135 143.

[20] P.C. Warterman, R.Truell.Multiple Scattering of Waves [J]. J.Math.Phys. Vo12. 1961. P512 540.

[21] Xu Zhiqiang, Chong Liqin, Wang Weidong. Coal Water Mixture Preparation Technology and Application in Replacing Oil to Generate Electricity. APPEEC 2009 Conference,2009,3.

[22] Wang Weidong, Tian Jinyun, Zhang Chenglian, Xu Zhiqiang. Study of coal water slurry Particle Size Distribution with Ultrasonic Testing Theory[J]. APPEEC 2010 Conference,2009,3.

[23] U Riebel. The fundamentals of particle size analysis by means of ultrasonic spectrometry [J], Particle and Particle Systems Characteisation,1989,6,135 143.

[24] V. Twersky.On scattering of waves by random distributions. I. Freespace scatterer formalism [J]. J. Math. Phys. 3, 700 715, 1962.

[25] Michael Baudoin, Jean-Louis Thomas, François Coulouvrat and Daniel Lhuillier.An extended coupled phase theory for the sound propagation in polydisperse concentrated suspensions of rigid particles [J]. 2007 Acoustical Society of America. 